\title{
Telemonitoramento como estratégia de cuidado longitudinal a grupos prioritários em tempos da COVID-19: uma experiência na atenção primária à saúde do município de Vitória-ES
}

\author{
Alana Pereira Rodrigues, Carla Rosa Felipe, Denise Bussú Lima, Luana Roberta Oliveira Costa, \\ Patrícia Felisberto Fernandes, Rhaviny de Paula Pêgo Silva, Rivana Marília Fernandes, \\ Welington Serra Lazarini
}

\section{RESUMO}

Com a pandemia da COVID -19, a atenção primária em saúde sofreu alterações tanto físicas quanto logísticas em todo o mundo para adaptar-se a essa situação emergencial. Enquanto os esforços dos gestores do sistema de saúde se orientaram prioritariamente para a organização dos serviços para a recepção dos casos suspeitos da COVID-19, o presente artigo relata uma estratégia de telemonitoramento dos pacientes que necessitam de acesso aos cuidados programados. Organizados em grupos prioritários pela equipe, o trabalho é realizado pelos Agentes Comunitários de Saúde, com suporte do Núcleo de Apoio de Saúde da Família. Adotada com o objetivo de garantir acesso ao cuidado, recursos e informações de saúde para a população de quase 12 mil habitantes atendidas por uma Unidade de Saúde do município de Vitória/ES, a iniciativa tem mostrado resultados satisfatórios.

Palavras-chave: Infecções por Coronavírus; Pandemias; Continuidade de Assistência a Paciente; Prioridades em Saúde; Atenção Primária à Saúde.

\section{ABSTRACT}

Due the COVID -19 pandemic, around the world to adapt to this emergency situation, the primary health care has undergone both hysical and logistical changes. While the efforts of health system managers were primarily oriented towards the organization of services to recieve suspected cases of COVID-19, this article reports a strategy for telemonitoring patients who need access to programmed care. It was organized priority groups by the team, the work is carried out by Community Health Agents, with support from the Family Health Support Center. Taking actions with the objective of to ensure to care, resources and health information for the population of almost 12 thousand inhabitants watched for a Health Unit in the municipality in Vitória/ES, the initiative has showed satisfactory results.

Keywords: Coronavirus Infections; Pandemics; Continuity of Patient Care; Health Priorities; Primary Health Care.

Submissão recebida em 30 de maio de 2020.

Aceito para publicação em 08 de junho de 2020.

Avaliado pelo sistema Double Blind Review com participação dos editores.
Revista da Rede APS 2020

Publicada em: 09/06/2020

DOI:10.14295/aps.v2i2.96

Alana Pereira Rodrigues

Denise Bussú Lima

Patrícia Felisberto Fernandes

Rivana Marília Fernandes

(Prefeitura Municipal de

Vitória, Vitória, ES, Brasil)

Carla Rosa Felipe

Luana Roberta O. Costa

Rhaviny de Paula P. Silva

(Instituto Capixaba de Ensino,

Pesquisa e Inovação em Saúde, Vitória, ES, Brasil)

Welington Serra Lazarini (Universidade Federal do Espírito Santo, Vitória, ES, Brasil)

Correspondência para: Alana Pereira Rodrigues alanapr@gmail.com

Carla Rosa Felipe carlarosafelipe@yahool.com.br

Welington Serra Lazarini welingtonsl@hotmail.com 


\section{INTRODUÇÃO}

A COVID-19 teve seus primeiros casos registrados no final de 2019, na província de Hubei, China. Trata-se de uma nova cepa da família Coronaviridae (Sars-Cov-2), cujo alto poder de transmissão propiciou um rápido avanço para todos os continentes nos meses seguintes, o que levou a Organização Mundial de Saúde (OMS) a declarar estado de pandemia da COVID-19 no dia 11 de março de 2020. Até o dia 28 de maio de 2020 foram confirmados mais de 5,5 milhões de casos e quase 354 mil mortes em decorrência da infecção pela Sars-Cov-2 no mundo (WHO, 2020).

Para além dos problemas sanitários provocados pela COVID-19, os impactos gerados pela pandemia nos âmbitos político, econômico, social e cultural fizeram com que vários chefes de Estado adotassem medidas de alto impacto, como as estratégias de isolamento social, na tentativa de conter a velocidade do curso de disseminação da COVID-19. Atrelado a isso, a gravidade da doença levou muitos desses países a uma corrida pela disponibilização de leitos de terapia intensiva (SARTI et al., 2020).

Embora a preocupação de parcela considerável da população, bem como os holofotes de grande parte da mídia, tenha se dirigido a estruturação e garantia de acesso ao ambiente hospitalar, fazse necessário repensar a organização dos serviços de atenção primária à saúde (APS) no contexto da pandemia da Covid-19, uma vez que estudos apontam que $80 \%$ dos casos são leves ou moderados (DUNLOP, HOWE, ALLEN, 2020).

A experiência relatada deu-se em uma Unidade de Saúde da Família (USF) localizada em Vitória, no Espírito Santo. Abrange em seu território quatro bairros da periferia do município que totalizam 11.855 habitantes, segundo o IBGE (2010). Abriga um quantitativo importante de pessoas em situação de vulnerabilidade, decorrente da baixa renda familiar, da violência e do tráfico de drogas presente nessa localidade.
A USF recebe o Programa de Residência Multiprofissional em Saúde da Família, que visa formar profissionais de saúde qualificados a responder às reais necessidades de saúde de usuários, famílias e comunidade, bem como contribuir para a construção de novos paradigmas de assistência à saúde, ampliando a resolutividade das equipes de saúde da família. O Programa de Residência Multiprofissional em Saúde da Família compõe o Núcleo de Apoio à Saúde da Família (NASF), que é uma das iniciativas implantadas no Brasil com o objetivo de fortalecer a APS. Os NASF são constituídos por profissionais de diversas especialidades que atuam na perspectiva do apoio matricial junto com as Equipes de Saúde da Família propiciando a efetivação de um arranjo assistencial mais abrangente e resolutivo no âmbito da APS, conforme estabelecido pelo Ministério da Saúde (BRASIL, 2009).

Assim, tem-se que, mesmo com as mudanças trazidas pela COVID-19, a APS precisa garantir o acesso à saúde e uma cobertura da população do território de forma integral e equânime (BRASIL, 2020). O objetivo da ação relatada é agir ativamente, no sentido de evitar que os usuários fiquem desassistidos e recebam um acompanhamento de qualidade mesmo diante da pandemia. Mantendo o acompanhamento dos usuários, objetiva-se possibilitar que a equipe médica e de enfermagem receba uma demanda mais qualificada, otimizando suas agendas para $\mathrm{o}$ atendimento dos pacientes sintomáticos respiratórios.

Os agentes comunitários de saúde ( $A C S^{\prime}$ s) têm um papel fundamental dentro desse processo, uma vez que suas atribuições específicas incluem: orientar famílias quanto à utilização dos serviços de saúde disponíveis; desenvolver atividades de promoção da saúde, de prevenção das doenças e de agravos e de vigilância à saúde por meio de visitas domiciliares e de ações educativas individuais e coletivas nos domicílios e na comunidade. Fazendo o elo com a equipe de saúde da família, visa mantê-la informada, principalmente a respeito das situações de risco e acompanhar o processo de desenvolvimento 
P.; Fernandes, R. M.; Lazarini, W. S.

desses agravos, de acordo com as necessidades definidas pela equipe (BRASIL, 2017).

\section{DELINEAMENTO DO PROCESSO: A}

\section{CONSTRUÇÃO DE NOVOS CAMINHOS}

Diante da pandemia do novo coronavírus, a equipe de saúde da família se deparou com um grande desafio. Era necessário realizar a escuta qualificada das necessidades dos usuários, proporcionando atendimento humanizado e viabilizando a manutenção do vínculo com a USF. Nesse sentido, foi desenvolvido um projeto de atendimento remoto via telefone pelos ACS's e os membros do NASF, com o apoio das equipes de saúde da família da unidade.
Tal estratégia se mostrou de extrema importância para a comunidade, muito carente de atendimento. Desse modo, esse projeto visou ofertar para a população orientações sobre a COVID-19 de maneira ativa, como medidas de prevenção, sinais e sintomas, bem como sobre os fluxos de funcionamento da USF durante a pandemia. Além disso, importou também abordar as epidemias de arboviroses em curso no território, que adquiriram um caráter de esquecimento pelos moradores. A ideia central do projeto é manter o elo entre usuários e USF, trabalhando sempre para promover a saúde e prevenir agravos junto à população. O fluxograma do trabalho realizado (Figura 1), bem como as quatro etapas que caracterizam sua organização serão apresentados a seguir.

Figura 1 - Fluxograma das etapas de reorganização do processo de trabalho para o acompanhamento longitudinal dos grupos prioritários em uma unidade de saúde da família em Vitória-ES.
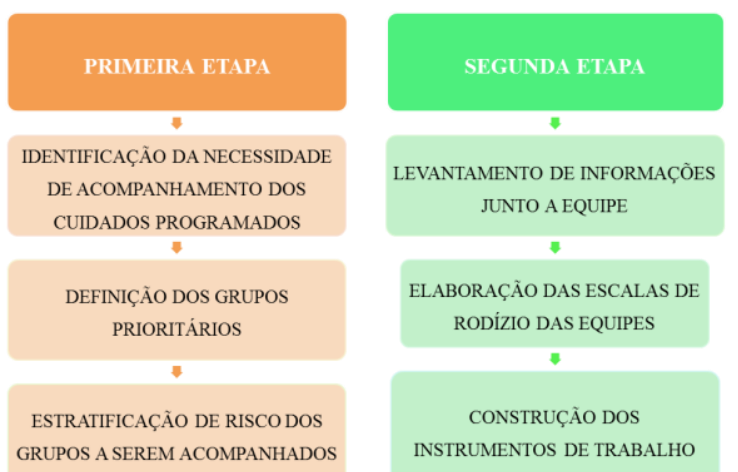
RODIZIO DAS EQUIPES

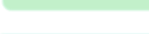

CONSTRUČ̃̃ DOS INSTRUMENTOS DE TRABALHO
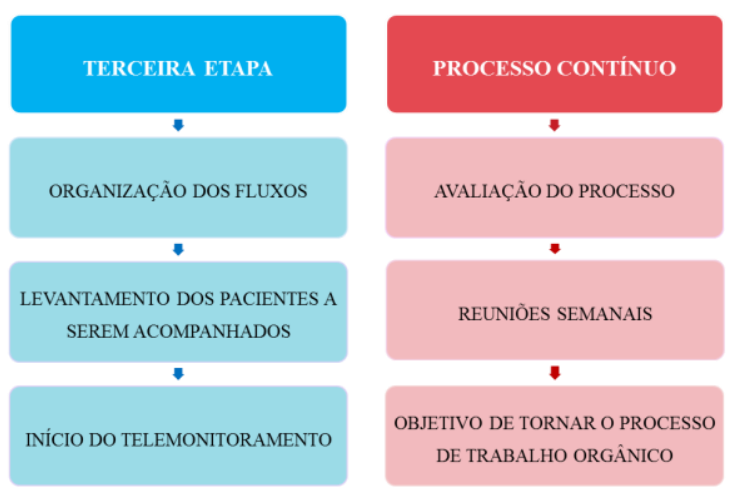

OBJETIVO DE TORNAR O PROCESSO DE TRABALHO ORGÂNICO

Fonte: Elaborado pelas autoras.

\section{Primeira etapa: identificação da \\ necessidade de acompanhamento dos cuidados programados}

Em reunião do NASF, após a suspensão das agendas eletivas de todos os profissionais e das visitas domiciliares de rotina dos ACS, por meio do decreto municipal (PREFEITURA MUNICIPAL DE VITÓRIA, 2020) a equipe entendeu a necessidade de garantir atendimento aos usuários que necessitam de acompanhamento e não apresentam quadro de síndromes gripais para quem o serviço foi priorizado naquele momento - endossando que essa população não deveria ficar desamparada pela USF. Em equipe, foram identificados em 11 grupos que necessitariam de manter o cuidado de uma forma programada, sendo eles: hipertensos, diabéticos, dialíticos, idosos, acamados, crianças de 0 a 2 anos, pacientes notificados por 
arboviroses, pacientes de saúde mental, gestantes, puérperas e recém-nascidos. Além disso, destacou-se também a importância de manter o acompanhamento dos pacientes de rotina, na medida em que se tornasse possível. Esses grupos também foram avaliados em uma estratificação de risco para a COVID-19 em que foram ressaltados os grupos prioritários para o acompanhamento.

\section{Segunda etapa: produção dos instrumentos de trabalho}

Ficou definido então que os ACS's, impossibilitados por decreto de realizar suas visitas domiciliares, realizariam o acompanhamento das famílias de maneira remota, por meio de ligações telefônicas, sendo acompanhados pela equipe do NASF, que ofereceria suporte na resolução das demandas trazidas pela equipe. Com o intuito de organizar o novo fluxo de trabalho, na primeira semana foram criadas escalas de trabalho que viabilizaram o home office para parte da equipe, enquanto a outra parte trabalhava presencialmente em esquema de rodízio, garantindo assim uma menor circulação de servidores dentro do serviço e, consequentemente, a proteção dos servidores e dos usuários. Para o acompanhamento ser efetivo foram criados três instrumentos visando ofertar suporte e garantir a longitudinalidade nesse novo modelo de cuidado, pautado no telemonitoramento. Foi construído coletivamente um roteiro para a realização dos contatos telefônicos, que contempla perguntas acerca da condição geral do paciente, existência de sintomas gripais, sinais de alarme e orientações específicas para cada um dos grupos prioritários supracitados. Nesse roteiro também foram inseridos os prazos para o próximo contato telefônico, que varia de acordo com o grupo e orientações gerais acerca da COVID-19 e os novos fluxos da USF.

A partir do conteúdo desse roteiro, foi construída uma série de 12 planilhas de acompanhamento, uma para cada um dos grupos prioritários, elaboradas no Microsoft
Excel $^{\circledR}$, com espaço para registro manual das informações coletadas durante a ligação telefônica e que sejam de interesse para monitoramento de cada um dos grupos descritos. As tabelas foram impressas e reprografadas com o intuito de permitir o registro e o seguimento dos dados coletados e dinamizar a supervisão do trabalho por parte da equipe do NASF.

Por fim, os ACS's receberam um arquivo com modelos para a evolução dos prontuários dos pacientes que são monitorados. Esse modelo é composto com as principais informações coletadas, de forma a otimizar o processo de registro e garantir o acesso de toda a equipe às informações primordiais de cada caso.

\section{Terceira etapa: organização dos fluxos de trabalho}

Após definição dos grupos para os cuidados programados, foi realizado, junto às equipes de saúde da família, um levantamento dos pacientes pertencentes a cada uma das condições referidas. Para este passo os dados foram coletados através da Rede Bem Estar que é o sistema eletrônico de saúde do município, por meio da condição referida registrada na ficha $A$ de cada paciente.

De posse das listagens, cada ACS iniciou os contatos com os pacientes de sua microárea. A equipe do NASF ficou responsável de acompanhar o processo de perto, ficando disponível para mediar as dúvidas e inquietações trazidas tanto com relação ao novo processo de trabalho, quanto a encaminhamento de discussão de casos bem como os novos fluxos e estratégias da USF. Com o estabelecimento deste novo processo de trabalho, houve a necessidade de adaptação de toda a equipe da USF. A experimentação possibilitou também o levantamento de pontos a serem trabalhados para que o processo fosse aprimorado. 


\section{PROCESSO CONTÍNUO: ALINHAMENTO}

\section{COLETIVO DO TRABALHO}

Em seu primeiro mês de atividades, a utilização das tabelas e experimentação das novas rotinas pela equipe, despertaram diversos apontamentos para melhoria dos instrumentos de trabalho construídos. A equipe NASF,

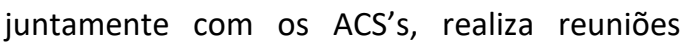
periódicas para avaliação e planejamento do processo de trabalho. Nestas reuniões sistemáticas são promovidas constantes atualizações dos instrumentos, com a realização de ajustes das planilhas de acompanhamento dos cuidados programados, a partir das discussões levantadas, com vistas a atender as questões trazidas durante o processo de telemonitoramento pelos ACS's e a supervisão da equipe NASF.

As tabelas preenchidas pelos ACS's, durante as ligações, são posteriormente analisadas e consolidadas pela equipe NASF em um arquivo único, que permite a equipe monitorar o trabalho, ofertando dados concretos para avaliação e planejamento dos próximos passos. Importante salientar também o dinamismo intrínseco aos processos de trabalho em saúde, em que as necessidades de saúde da população, ao serem ouvidas pela equipe, também apontam novos rumos para o delineamento do trabalho. Esse procedimento faz com que o projeto apresente maior capacidade de resposta às demanas de saúde da população, ainda que se encontre em momento de construção, uma vez que é um processo novo e que necessita se tornar orgânico junto à equipe.

\section{REORGANIZAÇÃO DO PROCESSO DE}

\section{TRABALHO: ALGUNS RESULTADOS}

\section{ALCANÇADOS}

O projeto se desenhou a partir da necessidade de acompanhamento sistemático de grupos prioritários durante a pandemia da COVID-19. Se, no primeiro momento, os serviços de saúde do município se concentraram nos cuidados aos pacientes sintomáticos respiratórios, o cancelamento das agendas eletivas, bem como a suspensão das visitas domiciliares de rotina, acendeu a necessidade de garantir a longitudinalidade do cuidado.

Com o início abrupto no mês de abril de 2020 frente à necessidade de responder ao desafio de acompanhar a população vinculada à USF, foram realizados pela equipe 540 contatos no período de 13 de abril, quando se iniciou a terceira etapa, ao dia 22 de maio desse ano (Figura 2). Esse período inicial de implantação também apontou algumas fragilidades, principalmente relacionados a dificuldade dos profissionais com o uso das tecnologias e ao preenchimento das fichas. Do total de contatos foram excluídos 23 registros, que apresentaram dados incompletos, letras ilegíveis ou informações que não se equalizavam com o processo de trabalho delineado, fazendo com que a equipe optasse em excluir do consolidado final apresentado neste artigo. 
Figura 2 - Distribuição do total de contatos telefônicos realizados por grupo prioritário entre 13 de abril a 22 de maio de 2020 em uma unidade de saúde da família, Vitória-ES.

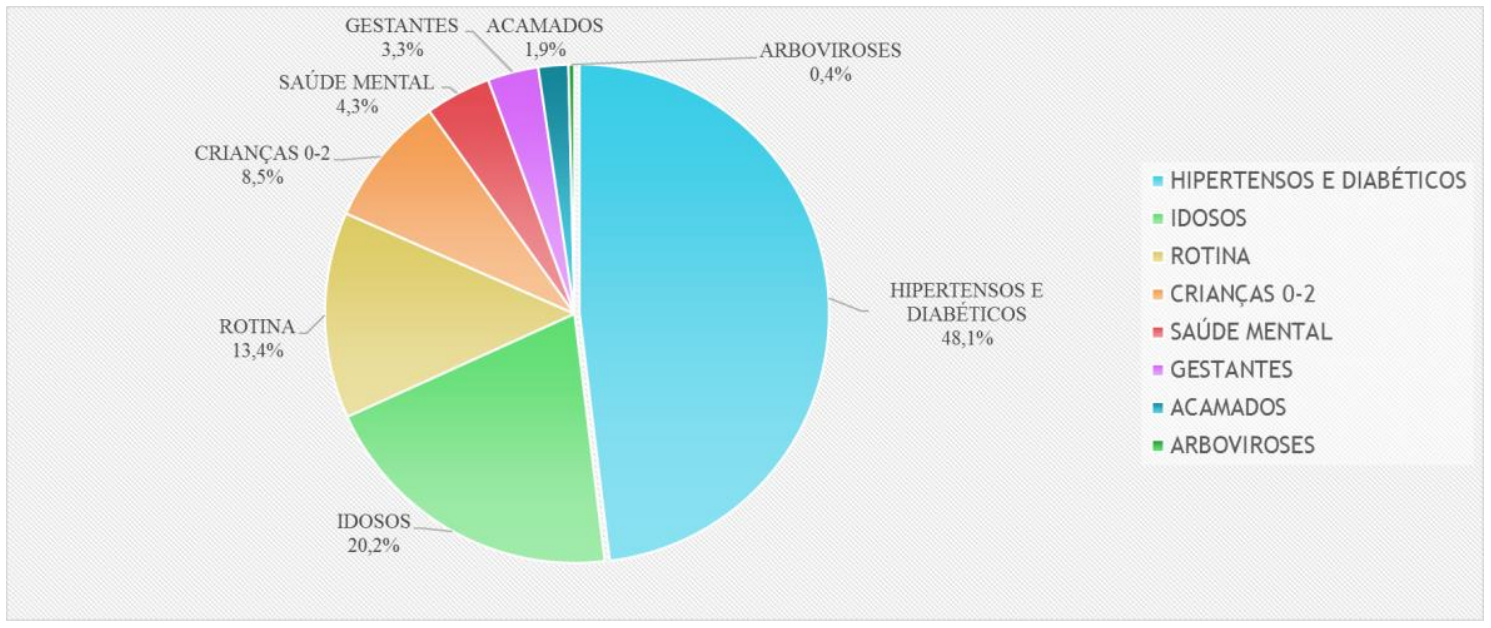

Fonte: Elaborado pelas autoras.

Desses usuários contactados, o público que obteve maior abrangência, foram os hipertensos e diabéticos, totalizando uma marca de $48,0 \%$ dos contatos realizados, seguido pelos idosos, que somaram $20,1 \%$ do total de contatos. Destaca-se, que ambos os grupos se caracterizam como risco elevado para as formas mais graves da COVID-19 e foram priorizados pela equipe na fase inicial do projeto com o intuito de proporcionar que os pacientes recebessem orientações adequadas, garantindo assim que suas condições crônicas estivessem controladas e acompanhadas.

Entre todos os contatos realizados, 14,0\% apresentaram alguma queixa e $8,9 \%$ apresentaram relatos de sintomas gripais. Esses receberam orientações sobre isolamento social, sobre quando procurar a USF ou pronto atendimento para atendimento presencial e sobre a possibilidade de atendimento médico remoto na central municipal de telemedicina, implantada durante a pandemia. Ressalta-se, que aos pacientes acamados que apresentaram sintomas gripais foi viabilizado visita domiciliar da equipe médica e de enfermagem para avaliação.
Quanto ao acesso às medicações, $11,3 \%$ dos pacientes estavam com situações que necessitavam de avaliação para renovação de prescrição medicamentosa, sendo orientados quanto ao novo fluxo de atendimentos da USF e, assim, possibilitando a otimização da ida do paciente ao serviço e diminuindo sua exposição aos riscos da COVID-19. Além destes, 13,0\% apresentaram aos ACS a demanda de necessidade de consulta médica presencial, sendo repassada e discutida com a equipe no intuito de construir estratégias que viabilizassem a oferta de cuidado no contexto da pandemia.

Destaca-se ainda que $10,4 \%$ dos contatos não foram concluídos, ou seja, não obtiveram sucesso, pois as ligações não foram completadas. Os motivos variaram entre caixa postal ou telefone não existente. Esse número elevado evidencia uma desatualização nos cadastros dos pacientes e sinaliza para a equipe a urgência do serviço de aproximar-se dos usuários também de forma remota, a partir da oferta de um maior acesso à tecnologia para a equipe.

Vale ainda ressaltar que o telemonitoramento tem apresentado boa adesão junto aos usuários 
do território, fato esse que se deve ao vínculo fortalecido que os ACS's possuem com a população. Os pacientes que receberam as ligações referiram sentimento de bem estar associando ao fato de ter sido lembrado pela equipe durante a pandemia, contribuindo assim para a diminuição do estresse causado pela sensação de solidão provocado pelo fechamento dos serviços e do isolamento social.

Outro dado importante registra que, de 25 profissionais da equipe envolvida nesse processo descrito, 2 encontram-se em regime total de home office, por encontrarem-se em grupo de risco para a COVID-19 e 10 foram afastados por licença médica em algum período durante o tempo de execução do projeto. Esses afastamentos e a consequente baixa na equipe em serviço coloca mais um desafio frente o acompanhamento da população e a reorganização do serviço de saúde diante da pandemia da COVID-19.

\section{CONSIDERAÇÕES FINAIS}

Considerando a atual pandemia da COVID-19 e a suspensão das atividades coletivas, atendimentos eletivos e consultas de rotina, urgiu a necessidade de reorganização do fluxo de trabalho na APS, de maneira que esse trabalho realizado pelo NASF, em conjunto com os ACS's, está sendo fundamental para manter a assistência à comunidade. Com o foco dos atendimentos presenciais voltados para os casos da COVID-19, o trabalho de acompanhamento dos grupos estratificados como prioritários, através do telemonitoramento, tem contribuído, de maneira ímpar, na garantia de assistência e oferta de informação com maior segurança e eficácia, sem necessariamente haver comparecimento à USF, com vistas a evitar a contaminação e transmissão do novo coronavírus.

Acredita-se que não justifica prevenir, somente, disseminação, agravos e mortes referentes à COVID-19 e postergar as demais necessidades das outras situações de risco, como os pacientes com transtornos mentais graves, hipertensão, diabetes, idosos, acamados, gestantes, entre outros já mencionados no trabalho. Uma vez que, se a população não receber a assistência adequada, poderá sofrer consequências e, inclusive, vir a óbito por outras complicações de saúde. $O$ trabalho de telemonitoramento realizado na USF tem funcionado de maneira eficiente e se aperfeiçoado semanalmente. Entretanto, é necessário ponderar algumas fragilidades existentes no processo. 


\section{NOTAS E REFERÊNCIAS}

BRASIL. Ministério da Saúde. Departamento de Atenção Básica. Diretrizes do NASF: Núcleo de Apoio a Saúde da Família. Brasília: Ministério da Saúde, 2009. Disponível em: <https://bvsms.saude.gov.br/bvs/publicacoes/caderno_atencao_basica_diretrizes_nasf.pdf>. Acesso em: 15 abr 2020.

BRASIL. Ministério da Saúde. Portaria no 2.436, de 21 de setembro de 2017. Aprova a Política Nacional de Atenção Básica, estabelecendo a revisão de diretrizes para a organização da Atenção Básica, no âmbito do Sistema Único de Saúde (SUS). Brasília, DF: Ministério da Saúde, 2017. Disponível em: <http://189.28.128.100/dab/docs/publicacoes/geral/pnab.pdf>. Acesso em: 15 abr 2020.

BRASIL. Ministério da Saúde. Universidade Aberta do SUS. Atualização: orientações gerais ao paciente com COVID-19 na Atenção Primária à Saúde. Brasília: Ministério da Saúde, 2020. Disponível em: <https://www.unasus.gov.br/cursos/curso/46168>. Acesso em: 15 abr 2020.

DUNLOP, C.; HOWE, A; Li, D.; ALLEN, L. N. The coronavirus outbreak: the central role of primary care in emergency preparedness and response. BJGP Open, v. 4, n. 1, 2020. Disponível em: <https://doi.org/10.3399/bjgpopen20X101041>. Acesso em 03 Mai 2020.

PREFEITURA MUNICIPAL DE VITÓRIA. Secretaria Municipal de Saúde. Portaria no 015, de 20 de março de 2020. Dispõe sobre a adoção de medidas adicionais, de caráter temporário e emergencial, para o enfrentamento da Situação de Emergência de saúde pública, decorrente de Pandemia em razão de doença infecciosa viral respiratória - COVID-19. Diário Oficial do Município de Vitória, Vitória, ES, 20 mar. 2020. p. 1.

SARTI, T. D. et al. Qual o papel da Atenção Primária à Saúde diante da pandemia provocada pela COVID19?. Epidemiologia e Serviços de Saúde, Brasília, v. 29, n. 2, e2020166, 2020. Disponível em <http://dx.doi.org/10.5123/s1679-49742020000200024>. Acesso em: 03 Mai 2020.

WORLD HEALTH ORGANIZATION. Coronavirus disease (COVID-2019): situation report 129. Genebra: World Health Organization, 2020. Disponível em: <https://www.who.int/docs/defaultsource/coronaviruse/situation-reports/20200528-covid-19-sitrep-129.pdf?sfvrsn=5b154880_2>. Acesso em: 29 Mai 2020. 